\title{
Congélation d'émulsions : de la mayonnaise à la métallurgie
}

\author{
Sylvain Deville ${ }^{(1)}$ (sylvain.deville@univ-lyon1.fr) et Cécile Monteux ${ }^{(2)}$ \\ (1) Institut Lumière Matière (UMR 5306 CNRS, Université Claude Bernard Lyon 1), \\ 10 rue Ada Byron, 69622 Villeurbanne \\ (2) Sciences et ingénierie de la matière molle (UMR 7615 CNRS, \\ ESPCI, Sorbonne Université), ESPCI, 10 rue Vauquelin, 75231 Paris Cedex 05
}

Si congeler de la mayonnaise n'est pas recommandé,

cela pourrait toutefois nous aider

à comprendre la fabrication

d'alliages métalliques,

la cryopréservation des cellules

ou encore la congélation

des sols en hiver.

Nous nous intéressons ici au cas des gouttes d'huile

dans une émulsion, observées

par microscopie confocale

au cours de la congélation.

De nombreux phénomènes

physiques (transport, diffusion,

solidification, instabilités) prennent

place lors de ce processus,

offrant aux physicien.ne.s

un problème inédit aux multiples

ramifications. Ces études pourraient

améliorer notre compréhension

de plusieurs phénomènes

de solidification, naturels comme

technologiques.

\section{Quand des cristaux de glace rencontrent des objets}

Petit matin d'hiver en Auvergne. Dans le jardin, une brouette à moitié remplie d'eau par les averses fréquentes des derniers jours. Pendant la nuit, la température a chuté, et l'eau accumulée dans ce récipient improvisé a gelé. En s'approchant de plus près, on distingue de longues bulles étirées, semblables à d'éphémères étoiles filantes capturées par la glace (fig. 1). D'autres, plus grosses, ont conservé leur forme sphérique. Le sol sous la brouette, une terre argileuse saturée d'eau, est également congelé, et présente un étrange aspect : des petits trous lamellaires et parallèles débouchent en surface ${ }^{(a)}$, devenue alors semblable à une terre griffée par quelque animal de passage.

La chute nocturne de la température sur les monts du Livradois a entrainé une germination puis une croissance lente et progressive de la glace à partir de l'eau environnante. Dans la brouette, l'oxygène initialement dissous dans l'eau ne pouvant être accommodé dans le réseau cristallin de la glace est rejeté dans le liquide restant, où le taux d'oxygène dépasse alors la limite de solubilité. Au cours de leur croissance, les cristaux de glace se heurtent aux bulles ainsi formées, les repoussant tant bien que mal, avant de les piéger progressivement dans ces formes fantomatiques et allongées. Dans la boue sous la brouette, ce sont les particules d'argile, beaucoup plus nombreuses et petites, que la glace a rencontrées, les repoussant progressivement. Ces particules se retrouvent alors concentrées, piégées puis compactées entre les cristaux de glace. Quand le sol se réchauffe sous les rayons du soleil matinal, la glace fond, mais l'organisation des particules d'argile est conservée, conduisant à cette texture si particulière.

Dans ces deux cas, un front de solidification - l'interface eau/glace - se retrouve en interaction avec des objets de diverses natures, ici des bulles ou des petites particules d'argile. Ce phénomène, omniprésent naturellement dans les régions froides, se retrouve également dans quantité de procédés technologiques, de l'élaboration des matériaux pour l'obtention de solides poreux allégés contenant des bulles, à la biologie (cryopréservation des cellules), ou en géophysique (congélations des sols, des lacs, ou de la mer).

Les physiciens, mais également les chercheurs de nombreux autres domaines, se sont depuis un siècle attelés à comprendre, prédire et maitriser ces processus. L'interaction entre les objets - particules, bulles, gouttes, cellules, virus - et les cristaux en croissance peut être mise à profit pour conférer aux matériaux des propriétés recherchées. Mais la présence d'impuretés peut aussi être dommageable. Par exemple, la présence de carbone lors de la croissance de monocristaux de silicium 


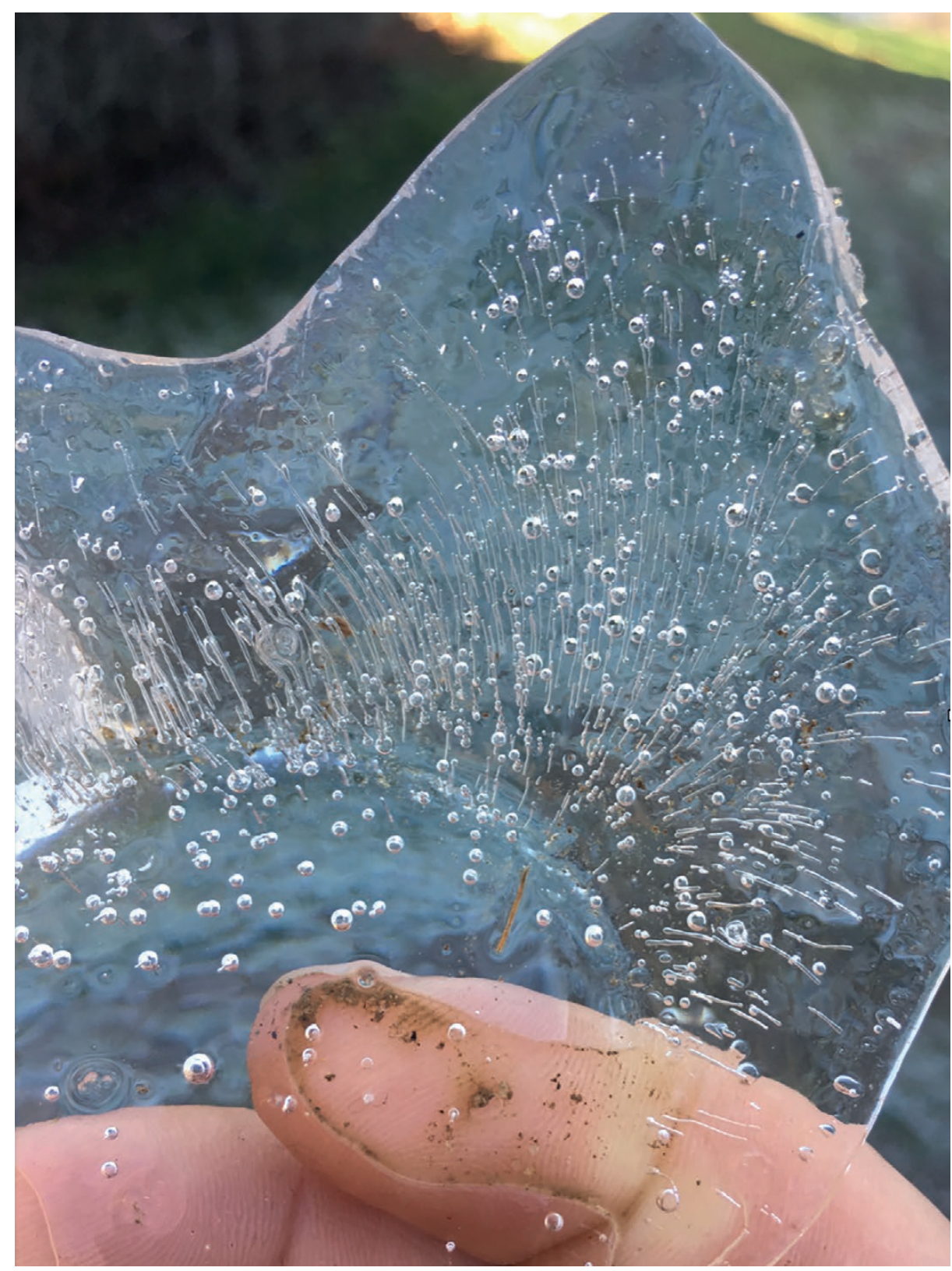

conduit à la formation de particules de carbure de silicium, qui peuvent être incorporées dans les monocristaux. La présence de ces particules peut alors dégrader les propriétés fonctionnelles des matériaux photovoltaïques ou de la microélectronique.

En première approximation, trois issues sont possibles lorsque des particules rencontrent un front de solidification : les objets peuvent être encapsulés directement, ou être rejetés pendant un certain temps ou indéfiniment par le front. Le comportement recherché lors de la solidification dépend fortement $\mathrm{du}$ contexte. La préparation d'alliages métalliques renforcés par des particules de céramiques requiert une distribution des particules la plus homogène possible, afin d'optimiser les propriétés mécaniques : une encapsulation immédiate est donc recherchée. De même, la production de matériaux cellulaires (mousses solides) exige de conserver la structure de la mousse lors de la solidification. Au contraire, le procédé de texturation par la glace, qui permet d'obtenir des matériaux à porosité anisotrope ultra résistants grâce à la croissance de cristaux de glace dans des suspensions colloïdales, requiert le rejet complet des particules. La croissance de monocristaux de silicium ou de saphir de haute qualité nécessite également une ségrégation complète des bulles et impuretés lors de la croissance.

1. Bulles d'air emprisonnées dans la glace par un froid matin d'hiver. Les doigts de l'un des auteurs donnent l'échelle. On notera que les plus grosses bulles ont été incorporées sans déformation, conservant leur forme sphérique, alors que les plus petites sont allongées, créant de véritables tunnels dans la glace dont la direction indique celle de l'avancée du front au cours de la congélation.

\section{Des modèles théoriques nombreux, mais encore trop simplistes}

Sous l'influence initiale des métallurgistes désireux de comprendre et maitriser les microstructures de solidification en présence de particules, les physiciens se sont depuis les années 1960 attelés à élaborer des modèles de plus en plus complexes pour décrire l'interaction entre une particule solide rigide isolée et un front de solidification en mouvement.
L'encapsulation d'une particule dans le solide nécessite de remplacer l'interface particule/eau par une interface solide/ particule. Un simple raisonnement thermodynamique sur la variation des énergies interfaciales avant et après encapsulation ne permet toutefois pas d'expliquer les comportements dynamiques observés lors de la congélation. De nombreux autres paramètres entrent en jeu dans la dynamique d'encapsulation, et raisonner en termes de forces appliquées sur les objets permet une meilleure analyse de ces phénomènes. 
$>>$

Une série de modèles théoriques prédit que lorsqu'un front de solidification se déplace et rencontre une particule immobile et isolée, plusieurs forces principales entrent en jeu : les forces d'interaction (van der Waals, force électrostatique, etc.) entre la particule et le front, et la friction visqueuse autour de la particule. L'équilibre entre ces forces conduit, en première approximation, à une vitesse critique du front (pour une taille donnée de la particule) au-delà de laquelle la particule sera encapsulée par le front. Inversement, pour une vitesse donnée, il existe une taille critique de particule au-delà de laquelle l'encapsulation est prédite.

La conductivité thermique de la particule joue un rôle important, modifiant localement le champ de température autour d'elle : ainsi, même si le gradient de température est fixé, une particule qui conduit mieux la chaleur que le liquide environnant implique une température plus élevée dans le film liquide entre la particule et le front de solidification, repoussant ainsi le front solide, qui devient donc concave (fig. 2a). À l'opposé, lorsque la particule conduit moins la chaleur que le liquide autour, le front solide s'avance davantage (fig. 2b).

La majorité des études s'est jusqu'à présent concentrée sur le cas d'objets rigides isolés dans un solvant pur, telles des particules céramiques dans un métal (liquide au départ, puis solidifié pour fixer leur distribution), où ces prédictions ont été validées de nombreuses fois. Bien que déjà compliqué, c'est pourtant le cas le plus simple ! En revanche, dans la majorité des situations, les objets sont nombreux, parfois agglomérés, et de tailles variables. La phase liquide est rarement pure, que ce soit par nature (solidification d'un alliage) ou par contamination (présence d'impuretés). La présence de solutés ou d'impuretés peut alors jouer un rôle essentiel, du fait de leur très faible limite de solubilité dans la glace. De forts gradients de concentration en soluté peuvent ainsi apparaitre près du front, modifiant localement la température de solidification.

Dans de nombreuses situations, ce sont des objets mous qui interagissent avec les cristaux en croissance, par exemple les bulles que l'on cherche à éliminer lors de la croissance de monocristaux, les gouttes d'huile pour la fabrication de crèmes glacées ou des cellules biologiques que l'on congèle pour les stocker. Le cas des particules déformables a été peu étudié. Des comportements encore plus riches sont attendus. Les bulles et les gouttes sont susceptibles de changer de forme lors de l'encapsulation dans le solide, telles nos bulles étirées puis piégées dans la glace de la brouette. La ségrégation des tensioactifs utilisés pour stabiliser les bulles et les gouttes peut générer des gradients de tension interfaciale ou de pression osmotique. Ce champ d'étude reste encore presque vierge à ce jour.

\section{Congélation d'émulsions : un phénomène complexe aux nombreuses ramifications physiques}

Nous avons récemment étudié par microscopie confocale ${ }^{(b)}$ la congélation d'émulsions : des gouttes d'huile isolées dans l'eau - un modèle expérimental relativement simple pour comprendre l'interaction entre des objets et un front de solidification. La dégustation des échantillons n'étant pas nécessaire, nous avons préféré une émulsion de benzoate de propyle dans l'eau à de la mayonnaise. Grâce à la microfluidique, il est très facile de produire des gouttes monodisperses et de taille contrôlée, la taille étant un paramètre clé contrôlant les interactions objets/front. La microfluidique permet aussi de faire des études sur un grand nombre d'objets et d'obtenir des mesures statistiques fiables. Dans notre cas, ce sont ainsi des centaines de gouttes dont le comportement peut être étudié dans chaque expérience.

La congélation de l'eau est un phénomène de solidification accessible expérimentalement et compatible avec l'utilisation de la microscopie confocale. Si les physiciens utilisent depuis longtemps certains alliages transparents comme analogues aux systèmes métallurgiques, le système eau/glace est caractéristique de nombreuses occurrences naturelles ou technologiques.

2. Interaction entre un objet, de conductivité thermique k, et un front de solidification en déplacement : impact de la différence de conductivité thermique entre l'objet et l'eau. Le front avance à une vitesse $\mathrm{V}$.

(a) Si l'objet est conducteur (particule de métal, par exemple), le front de solidification devient concave.

(b) Si l'objet est isolant thermiquement (une bulle de gaz par exemple), les isothermes sont déformées en direction de l'objet, modifiant les interactions entre l'objet et le front de solidification, et donc les microstructures de solidification. 
De plus, afin d'étudier le rôle des solutés sur l'interaction goutte/glace, nous avons utilisé des fluorophores à la fois dans l'huile et dans l'eau, ce qui nous a permis de quantifier simultanément le déplacement des gouttes d'huile et les gradients de concentration en tensioactif rejeté près du front de glace (voir l'encadré ci-contre sur les développements expérimentaux).

Le comportement des gouttes d'huile dans l'eau lors de la congélation conduit à une succession complexe de phénomènes, où la physique entre en jeu, couplant un changement de phase à des problèmes de stabilité, de transport, d'hydrodynamique, de physico-chimie, et de mécanique de la matière molle.

\section{Des gouttelettes s'enfuyant}

\section{à l'approche du front de solidification}

Première constatation et première surprise : les gouttes d'huile, initialement immobiles, sont repoussées par le front qui avance vers elles. Ainsi, à une distance de plusieurs fois le diamètre moyen des gouttes - soit jusqu'à plusieurs centaines de micromètres dans notre cas - celles-ci accélèrent progressivement pour atteindre une vitesse, $u$, d'environ $1 \mu \mathrm{m} / \mathrm{s}$. Cette vitesse se maintient sur une distance de plusieurs dizaines de microns. Ce mouvement a plusieurs conséquences majeures sur l'évolution du système. Si la vitesse de déplacement des gouttes à l'approche du front est du même ordre de grandeur ou plus faible que celle de l'avancée du front, $V$, qui vaut entre 1 et $10 \mu \mathrm{m} / \mathrm{s}$ dans notre expérience, les gouttes sont repoussées pendant plusieurs secondes avant d'être rattrapées par le front. Pendant ce temps, elles rencontrent d'autres gouttes, qui se mettent elles aussi en mouvement à la même vitesse. Lorsque l'interface rattrape enfin les gouttes, ce ne sont donc plus des gouttes isolées mais des agglomérats de gouttes qui interagissent avec le front (fig. 3). La friction visqueuse qui s'exerce sur ces agglomérats et freine leur mouvement de fuite, augmente au fur et à mesure que les agrégats grossissent, entraînant leur incorporation dans la glace. La distribution des objets dans la phase solide en est fortement impactée. Ce comportement pourrait nous aider à comprendre comment des objets initialement isolés peuvent se retrouver concentrés en poches dans la phase solide, alors que leur taille individuelle ne permettait pas de prédire un tel comportement avec les modèles physiques actuels.

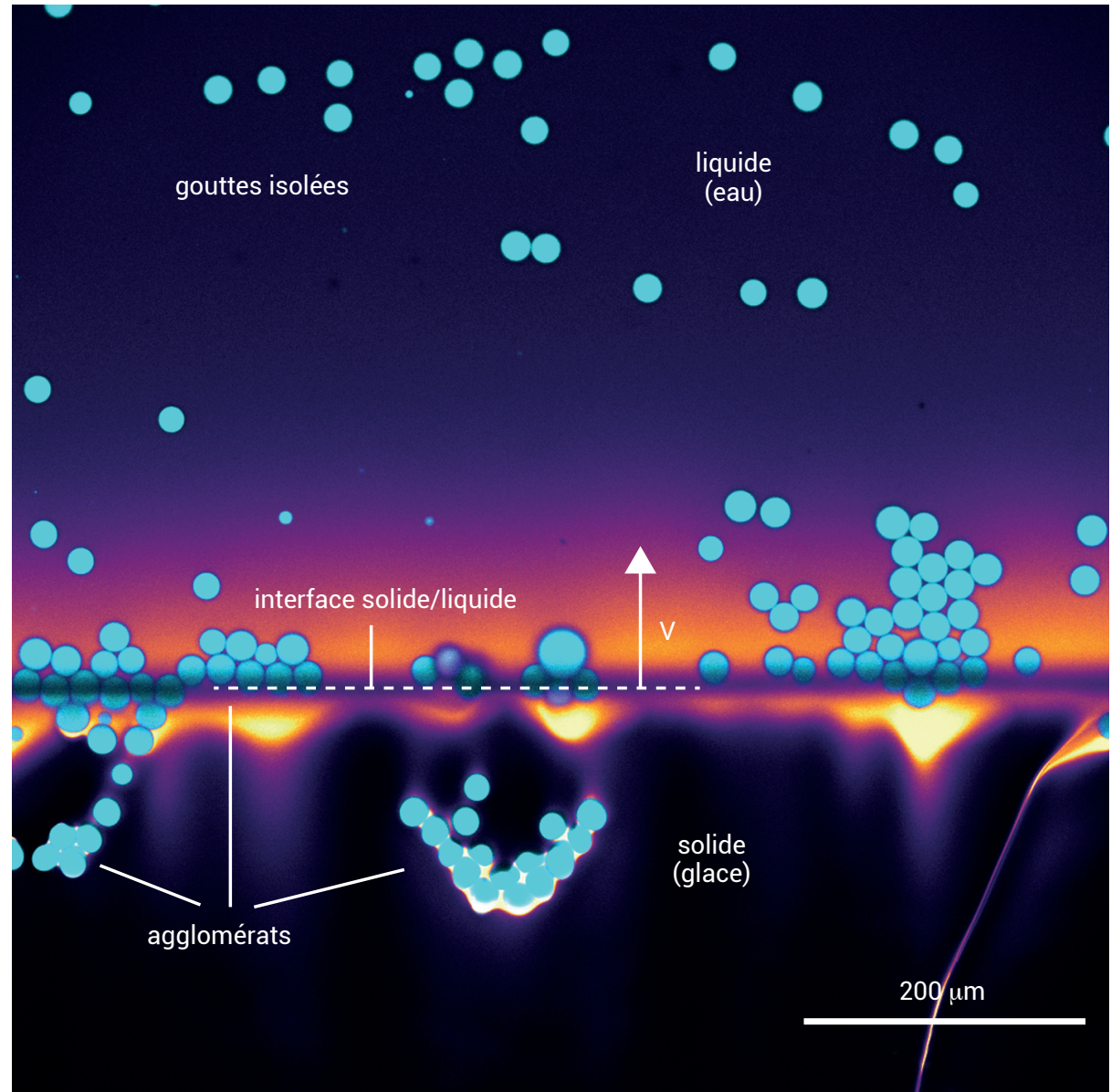

3. Formation d'agglomérats de gouttes à l'interface eau/glace, observée par microscopie confocale de fluorescence. À faible vitesse d'avancée du front, les gouttes sont suffisamment repoussées par le front pour former des agglomérats. De taille plus grande que les gouttes isolées, les agglomérats finissent par être incorporés dans la glace. La distribution spatiale des gouttes d'huile dans la phase solide s'en trouve donc fortement modifiée. Les gouttelettes d'huile sont marquées en bleu avec du BODIPY. De la rhodamine, fluorescente, est ajoutée dans l'eau, ce qui se traduit par un gradient de couleur allant du jaune au violet près de l'interface eau/glace. La rhodamine s'accumule près de cette interface, car elle est rejetée par la glace.

\section{Développements expérimentaux}

Si congeler reste simple, observer localement la congélation, à l'échelle des objets qui interagissent avec le front, l'est beaucoup moins. La mise au point du système, menée au LSPC (CNRS/Saint-Gobain) avec l'aide de Dmytro Dedovets, a soulevé plusieurs difficultés : nous avons dû choisir une huile et un tensioactif dont les points de congélation sont bien plus bas que celui de l'eau - afin d'éviter la congélation des gouttes et du tensioactif avant celle de l'eau - et d'une densité la plus proche possible de l'eau. Nous avons choisi comme huile le benzoate de propyle, et comme tensioactif le tween 80 (polysorbate 80 ), utilisé couramment pour la stabilisation d'émulsions. Pour rendre les gouttelettes d'huile fluorescentes, nous avons utilisé du BODIPY. Afin de suivre l'accumulation du tensioactif à l'interface eau/glace, nous avons ajouté dans la phase aqueuse de la rhodamine, un colorant dont le coefficient de diffusion est proche de celui du tensioactif et qui nous sert donc de jauge. La partie la plus difficile a toutefois été le développement d'une platine de congélation pour mener les expériences in situ sous le microscope confocal. Il est nécessaire d'isoler thermiquement la platine de l'extérieur afin de parfaitement contrôler la température de l'échantillon fixée à $-15^{\circ} \mathrm{C}$ et de limiter la condensation. De plus, la planéité du montage est cruciale, afin de garder le même plan d'observation pendant toute la durée de l'expérience, qui peut aller jusqu'à quelques heures, lorsque la vitesse de translation est de l'ordre du micron par seconde. 
$\gg>$

Un phénomène crucial semble être à l'origine du mouvement des gouttes : le rejet du tensioactif par les cristaux de glace lors de leur croissance conduit à l'instauration d'un gradient de concentration en tensioactif à l'interface eau/glace et influence le déplacement des gouttes. Ainsi, sur la figure 3 la couleur jaune/rose correspond à la rhodamine, molécule fluorescente ajoutée en très faible quantité dans l'eau. L'intensité de la fluorescence émise par la rhodamine dépendant de sa concentration, on observe ainsi que la glace, qui apparaît noire, rejette la rhodamine et qu'en conséquence celle-ci s'accumule à l'interface eau/glace. Le gradient de concentration en rhodamine à l'interface dépend de son coefficient de diffusion et de la vitesse du front. Le coefficient de diffusion du tensioactif étant égal à celui de la rhodamine, on en déduit que le tensioactif aussi s'accumule à l'interface eau/glace. Nous avons observé que la vitesse des gouttes augmente avec le gradient de concentration en tensioactif, suggérant qu'il joue un rôle essentiel sur l'interaction entre les gouttes et le front. Nous pensons que ce gradient de concentration modifie l'équilibre des forces s'exerçant sur la particule et induit une répulsion entre la particule et le front, par un phénomène appelé " diffusiophorèse ". Si ce phénomène de transport commence à être étudié dans des gradients de soluté bien contrôlés (en microfluidique par exemple), la présence de diffusiophorèse dans un phénomène de solidification semble nouvelle, et élargit donc le spectre des études.

\section{Une incorporation progressive par le front}

Deuxième étape du phénomène : l'incorporation par le front, lorsque celui-ci entre en contact avec les gouttes d'huile. La microscopie confocale et ses possibilités d'observations dynamiques nous offrent une vision unique de ces interactions. L'incorporation d'une goutte par le front est progressive et dynamique (fig. 4), et l'observation par fluorescence du gradient de soluté (tensioactif et rhodamine) à l'interface est riche en informations. Lorsque la goutte s'approche du front, la concentration en soluté augmente localement dans le film liquide entre la goutte et la glace, conduisant à une baisse locale de la température de congélation. Cela provoque une déstabilisation de l'interface eau-glace

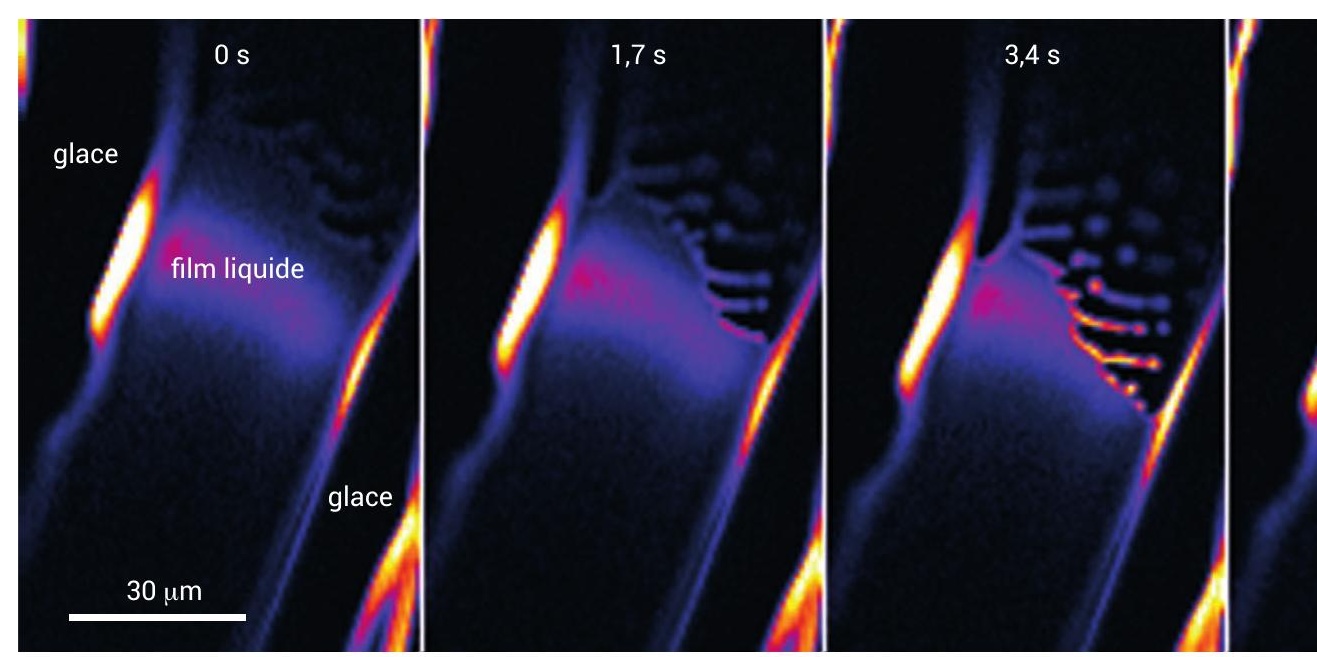

qui devient concave (fig. 4b). Une fois la goutte en partie encapsulée dans la glace, on observe derrière la goutte la formation d'un joint de grains (interface entre deux cristaux de glace adjacents), enrichi en soluté (fig. 4d). Cela veut donc dire que la présence des gouttes et du tensioactif modifie la taille des cristaux de glace et que les solutés (tensioactif et rhodamine) ont tendance à s'accumuler dans les joints de grains. Ceci fait écho à la tendance des particules dans un alliage à provoquer une diminution de la taille des cristaux et donc des grains, augmentant ainsi leur résistance mécanique (loi de Hall-Petch). Dans le cas des émulsions, la présence des gouttes peut donc aussi conduire à une déstabilisation du front plan de solidification, qui adopte alors une morphologie cellulaire, bien connue des physiciens étudiant la solidification d'alliages transparents.

À terme, ceci pourrait se révéler être un modèle intéressant pour les systèmes où des phases secondaires de compositions différentes peuvent être formées. La congélation d'émulsions permet en effet d'observer l'impact de la présence et l'agglomération de particules (ici des gouttelettes) sur la

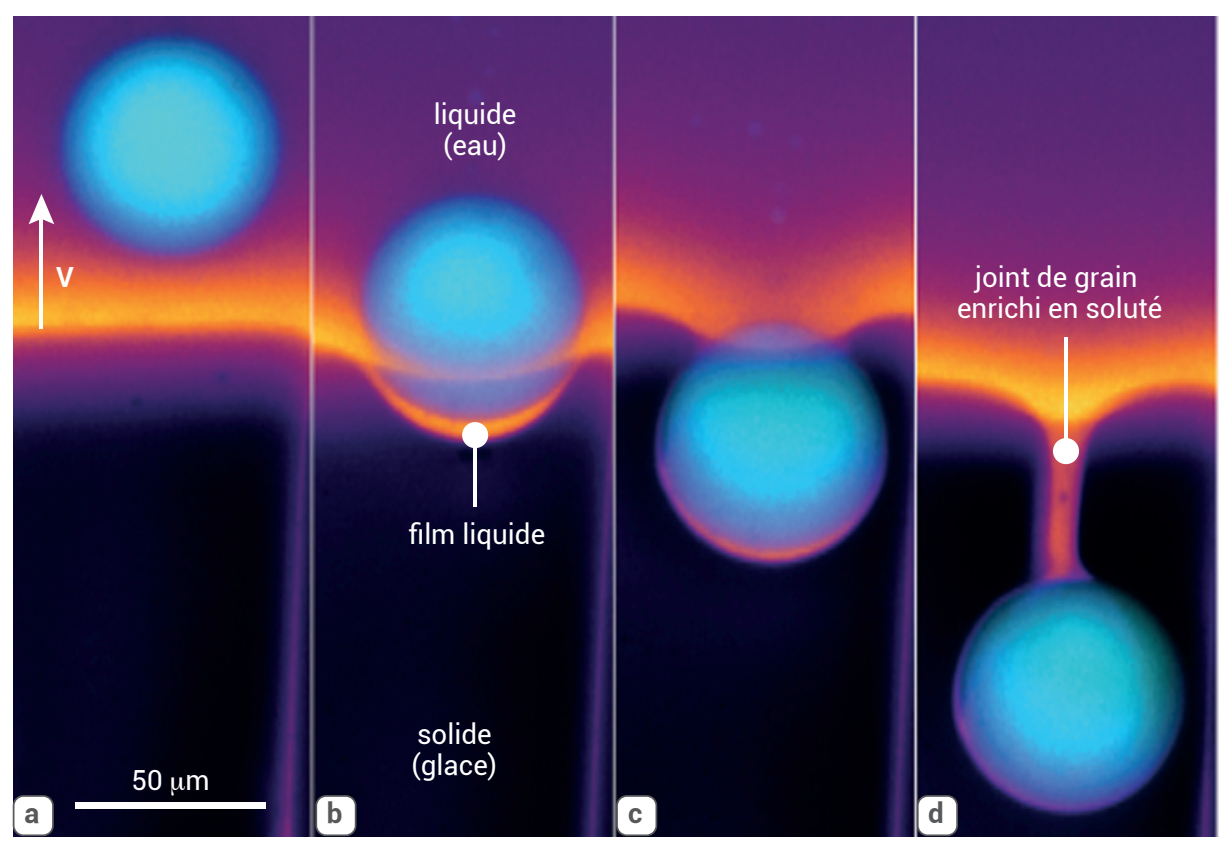

4. Séquence de l'incorporation d'une goutte d'huile isolée par le front de solidification (20 secondes entre chaque image). On notera la formation d'un film liquide enrichi en soluté entre la goutte d'huile et le front (b, c). Le film est conservé autour de la goutte une fois celle-ci incorporée dans la glace. La goutte crée dans son sillage un joint de grains enrichi en soluté (d), modifiant ainsi localement la microstructure du solide. 


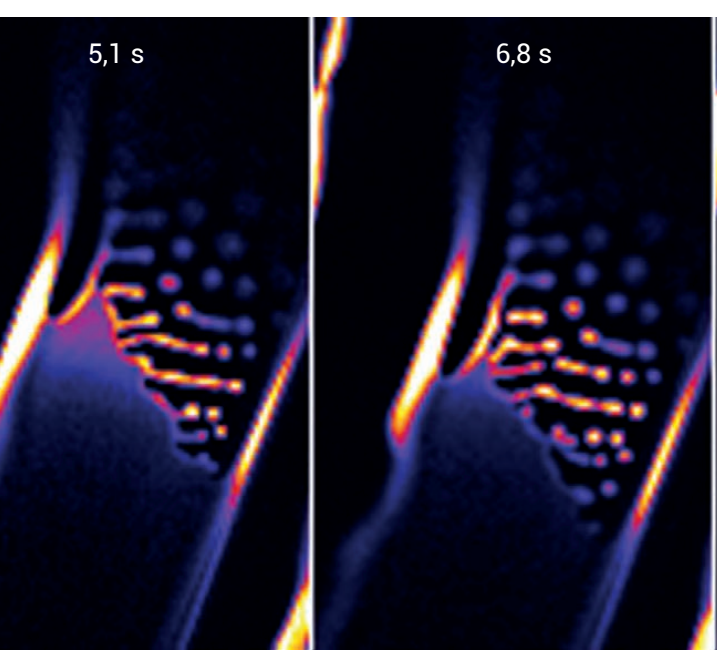

ségrégation du soluté et la formation de phases secondaires se solidifiant ultérieurement. Dans le cas de la cryopréservation, de nombreux additifs antigel sont ajoutés pour garantir la viabilité des cellules après congélation et décongélation. La concentration locale en soluté autour des cellules au cours des processus de préservation pourrait donc être beaucoup plus élevée que la concentration initiale en soluté ne le laisse présager. Une meilleure compréhension de la redistribution du soluté pourrait éventuellement envisager de réduire les concentrations d'additifs utilisés, avec à terme une meilleure préservation des cellules.

L'impact de la présence d'objets (particules ou bulles) sur la redistribution du soluté et des microstructures de solidification est un problème encore loin d'être résolu.

\section{Des films liquides se fragmentent en gouttelettes}

Nous avons observé un autre phénomène physique intrigant. La concentration locale en soluté, plus élevée aux joints de grains, permet à ces derniers de rester à l'état liquide à une température plus basse. Lorsque la solidification continue, avec la baisse de la température, ces films perdent de l'épaisseur et peuvent éventuellement se déstabiliser. Le film liquide plan et continu se transforme alors en une nappe de gouttelettes enrichies en soluté (fig. 5). Si les mécanismes physiques à l'œuvre restent encore à identifier, les fréquences élevées d'acquisition en microscopie confocale permettent de capturer en détail cette déstabilisation, qui se produit relativement lentement (en quelques dizaines de secondes).
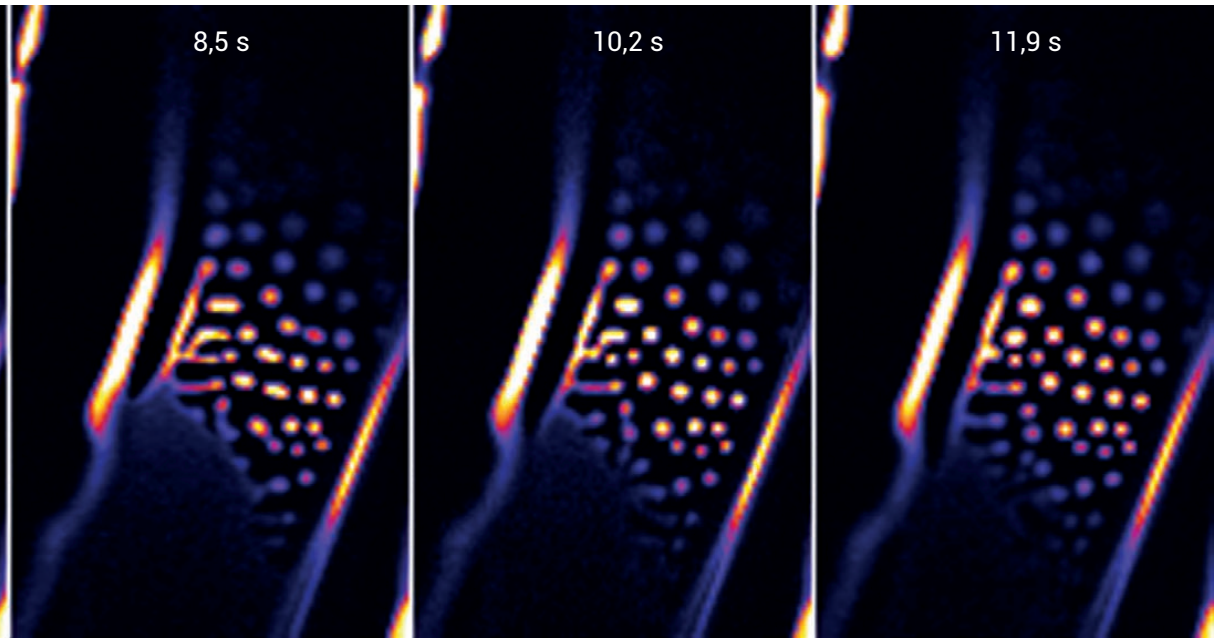

5. Séquence temporelle de la déstabilisation d'un film liquide enrichi en soluté entre deux

\section{De nombreuses questions ouvertes}

Notre étude a montré que le mouvement des gouttes d'huile, leur incorporation dans la glace, ainsi que la morphologie des cristaux de glace dépendent du phénomène de rejet des solutés par la glace, qui induit un fort gradient de concentration en soluté près de l'interface eau-glace. Impossible donc de garder la mayonnaise (émulsion vinaigre/huile) homogène si on la congèle !

Ce travail débouche sur de nouvelles questions : les émulsions sont-elles des analogues valides pour les alliages métalliques ? Quels sont les mécanismes de déstabilisation des films liquides aux interfaces entre les cristaux ? Le mouvement des gouttes en avant du front est-il dû à des mécanismes de diffusiophorèse ? La forte concentration locale en soluté peut-elle induire des dommages physiques aux objets encapsulés, notamment lorsque ceux-ci des cellules, par exemple - présentent une membrane permettant des échanges ? Les mystères de la congélation sont encore nombreux. cristaux en croissance. La croissance des cristaux réduit l'épaisseur du film en même temps que la concentration en soluté augmente. En dessous d'une épaisseur critique, le film se déstabilise progressivement en une nappe de gouttelettes. Puisqu'aucun soluté n'est incorporé dans la glace, la concentration en soluté dans les gouttelettes est plus élevée que dans le film initial (fluorescence plus importante).

\section{En savoir plus}

- D. Dedovets, C. Monteux et S. Deville, "Five-dimensional imaging of freezing emulsions with solute effects", Science. 360 (2018) 303-306

- Vidéo explicative sur YouTube accompagnant la publication de l'article, avec de nombreux extraits vidéo de l'étude : www.youtube.com/ watch?v=Ker7NbOwuxc

- "Crème très fraîche! ", épisode de Zeste de science, la chaine YouTube du CNRS, sur cette étude : www.youtube.com/ watch?v=SvjqBzxzNYs

(a) Voir par exemple les motifs de glace dans la boue gelée sur www.alamy.com/ice-pattern-in-frozen-glacial-mud-iceland-image241295949.html, ou https://claudiamcgillart.wordpress.com/2015/02/15/shopping-and-some-other-things/olympus-digitalcamera-855/

(b) La microscopie confocale est une technique de microscopie optique qui permet, par l'ajout d'un diaphragme, de ne sélectionner que la lumière provenant du plan focal observé. On élimine ainsi les rayons provenant des plans supérieurs et inférieurs au plan focal, ce qui améliore considérablement la résolution et la netteté des images. En déplaçant le plan focal observé, il est possible de reconstruire une image tridimensionnelle de l'échantillon. La source de lumière est un rayon laser, qui balaie l'échantillon ligne par ligne et excite la fluorescence des marqueurs incorporés. 